\title{
NONLINEAR CONTROL DESIGN OF ROBOTIC MANIPULATORS WITH VELOCITY OBSERVERS
}

\author{
Fang-Shiung Chen and Jung-Shan Lin
}

\author{
Department of Electrical Engineering \\ National Chi Nan University \\ 301 University Road, Puli \\ Nantou, Taiwan 545, R.O.C.
}

\begin{abstract}
At present, robotic manipulators are commonly used in manufacturing industry. In many cases, their end-effectors are required to move from one place to the other and follow some desired trajectories. This paper develops a nonlinear backstepping control design scheme with velocity observers for the trajectory tracking control of robotic manipulators. In fact, most of robot manipulator controllers require position as well as velocity measurements to achieve their control objectives, but a lot of robotic systems do not have the velocity measurement devices. Therefore, a velocity observer is designed to estimate the unknown angular velocity of robot manipulators, and then the nonlinear controller based on backstepping design algorithm is developed for the control of robot manipulator systems. The proposed nonlinear backstepping controller is not only to stabilize the robot system, but also to drive the trajectory tracking errors to converge to zero exponentially. Furthermore, some simulation results are given to illustrate the excellent performance of the backstepping control design scheme applied to a two-link robot manipulator. Copyright (C) 2005 IFAC
\end{abstract}

Keywords: Nonlinear control, Observers, Robotic manipulators

\section{INTRODUCTION}

In recent years, nonlinear and adaptive backstepping (Khalil, 2002; Krstić et al., 1995) control design schemes have been widely developed and applied in various nonlinear systems, including inverted pendulum systems (Tsai and Lin, 2003), active suspensions (Lin and Huang, 2003), antilock braking systems (Ting and Lin, 2004) and so forth. Those nonlinear and adaptive controllers could indeed achieve their desired control objectives excellently. For example, in (Tsai and Lin, 2003), a nonlinear backstepping design scheme was proposed to solve the problem of balancing a 360-degree inverted pendulum system by moving the cart horizontally. Here, the major target of this paper is to employ the backstepping control design methodology for the motion control of robotic manipulator systems. The control goal is to drive each link of a robot manipulator to track its own desired trajectory.

A great number of researches have been done for the trajectory tracking control of robot manipulators. Most of these existing controllers require the information of both position and velocity measurements for each link of robot manipulators, but lots of robot manipulator systems do not provide velocity measurements. Therefore, some research results have been developed for the link position tracking control without velocity measurements. In (Nisosia and Tomei, 1990), a 
velocity observer based on the angular position measurements and the system dynamic model was developed for robot manipulator control design. According to this paper, several different controllers have been developed in (Calugi et al., 2002; Eric and Lu, 1996; Lim et al., 1996). In addition, a robust tracking control law (Berghuis and Nijmeijer, 1991) was proposed with a linear observer which is simpler than the other previous observers.

In this paper, a nonlinear backstepping control design scheme with a velocity observer is proposed for the trajectory tracking control of a robot manipulator which has exact model knowledge. The exponential stability of the resulting closedloop system is demonstrated via Lyapunov stability theory with a useful lemma in (Wen and Bayard, 1988). This lemma is essentially a local stability theorem that establishes a region of convergence. Furthermore, the proposed control design scheme is applied to a two-link robot manipulator for comparative simulations. As a result, the proposed nonlinear backstepping control design is not only to stabilize the robot system, but also to force the tracking errors to converge to zero exponentially.

The remainders of this paper are organized as follows. In Section 2, the dynamic model of a robot manipulator is introduced with some important properties of the system. The velocity observer and the torque input controller are developed with backstepping design schemes in Section 3. In Section 4 , the stability of the system is investigated and analyzed via Lyapunov stability theorem and the useful lemma shown in Appendix. The simulation results are illustrated in Section 5, and some concluding remarks are given in Section 6 .

\section{SYSTEM MODEL AND DYNAMICS}

Derivation of the dynamic model of a robot manipulator plays an important role for analysis of manipulator structures, design of control algorithms, and simulation of motion. The dynamic equations of motion of a manipulator are a set of mathematical equations describing the relationship between the joint actuator torques and the motion of the structure. In this section, we will concentrate on the formulation, characteristics, and properties of the dynamic equations of robot manipulations for our control purposes.

In general, the dynamic model of an n-link rigidbody robot manipulator can be written in the following matrix equation (Craig, 1988):

$$
M(q) \ddot{q}+C(q, \dot{q}) \dot{q}+G(q)=\tau,
$$

where $q$ is the $n \times 1$ vector of generalized joint coordinates, $\tau$ is the $n \times 1$ vector of joint control input torques, $M(q)$ is the $n \times n$ positive definite symmetric inertia matrix, $G(q)$ is the $n \times 1$ vector of gravity forces, $C(q, \dot{q})$ is the $n \times n$ Corioliscentripetal matrix. From (1), the equation of motion can be rewritten as

$$
\ddot{q}=M(q)^{-1}[\tau-C(q, \dot{q}) \dot{q}-G(q)]
$$

In this system, it is assumed that only the measurement of the position vector $q$ is available. The control algorithm will be designed based on some very important properties of system model which are given as follows:

Property 1: The inertia matrix $M(q)$ is a symmetric positive definite matrix which verifies

$$
m_{m}\|x\|^{2} \leq x^{T} M(q) x \leq m_{M}\|x\|^{2}, \quad \forall x \in \Re^{n},
$$

where $m_{m}$ and $m_{M}$ denotes the strictly positive minimum and maximum eigenvalues of $M$ for all configurations $q$.

Property 2: The matrix $\dot{M}(q)-2 C(q, \dot{q})$ is skewsymmetric, that is, for any $x \in \Re^{n}$ and $x \neq 0$, we have

$$
x^{T}[\dot{M}(q)-2 C(q, \dot{q})] x=0 .
$$

Property 3: For $x, y \in \Re^{n}$, we have

$$
C(q, x) y=C(q, y) x \text {. }
$$

Property 4: The matrix $C(q, \dot{q})$ satisfies

$$
\|C(q, \dot{q})\| \leq c_{M}\|\dot{q}\|,
$$

for some bounded constant $c_{M}>0$.

\section{BACKSTEPPING DESIGN WITH VELOCITY OBSERVER}

In this section, it is assumed that only the measurement of position vector $q$ is available. A nonlinear backstepping control law with a linear velocity observer is proposed for the trajectory tracking control of robot manipulators. Now we employ backstepping schemes to design the nonlinear controller and observer. The backstepping design procedure consists of two steps:

STEP 1: The regulated variable is selected as

$$
z_{1}=q-q_{d} .
$$

The derivative of $z_{1}$ is calculated as

$$
\dot{z}_{1}=\dot{q}-\dot{q}_{d} \equiv x,
$$

and $x$ is used as the first virtual control variable. We choose the following stabilizing function as

$$
\alpha_{1}=-\Lambda_{1} z_{1}
$$


where $\Lambda_{1}$ is an $n \times n$ positive definite matrix with $\lambda_{m} I \leq \Lambda_{1} \leq \lambda_{M} I$, where $\lambda_{m}$ and $\lambda_{M}$ are the minimum and maximum of eigenvalues of $\Lambda_{1}$. The corresponding error state variable is defined as

$$
z_{2}=x-\alpha_{1}=x+\Lambda_{1} z_{1}=\dot{q}-\dot{q}_{r},
$$

where $\dot{q}_{r}$ is defined as

$$
\dot{q}_{r}=\dot{q}_{d}-\Lambda_{1} z_{1} .
$$

Therefore, the resulting error equation (8) becomes

$$
\dot{z}_{1}=x=z_{2}+\alpha_{1}=-\Lambda_{1} z_{1}+z_{2} .
$$

STEP 2: The derivative of $z_{2}$ is computed as

$$
\begin{aligned}
\dot{z}_{2}= & \dot{x}-\dot{\alpha}_{1}=\ddot{q}-\ddot{q}_{d}+\Lambda_{1} \dot{z}_{1} \\
= & M^{-1}(q)[\tau-C(q, \dot{q}) \dot{q}-G(q)]-\ddot{q}_{d} \\
& -\Lambda_{1}^{2} z_{1}+\Lambda_{1} z_{2} .
\end{aligned}
$$

Finally, according to the Lyapunov stability analysis, the control input is designed as

$$
\begin{aligned}
\tau= & M(q) \ddot{q}_{d}+C(q, \dot{\hat{q}}) \dot{q}_{r}-z_{1} \\
& -K_{1}\left(\dot{\hat{q}}-\dot{q}_{r}\right)+G(q),
\end{aligned}
$$

where $\dot{\hat{q}}$ is the estimated velocity vector with

$$
\dot{\hat{q}}=\dot{q}_{d}+L_{d}(q-\hat{q}),
$$

and $K_{1}$ and $L_{d}$ are positive definite matrices with $k_{m} I \leq K_{1} \leq k_{M} I$ and $l_{m} I \leq L_{d} \leq l_{M} I$, and $k_{m}, k_{M}, l_{m}, l_{M}$ are positive.

\section{STABILITY ANALYSIS}

Now, consider the stability analysis of the resulting closed-loop system with the following Lyapunov function candidate:

$$
V=\frac{1}{2} z_{1}^{T} z_{1}+\frac{1}{2} z_{2}^{T} M(q) z_{2}+\frac{1}{2} \dot{\tilde{q}}^{T} M(q) \dot{\tilde{q}},
$$

where $\dot{\tilde{q}}=\dot{q}-\dot{\hat{q}}$ is the velocity estimation error vector. From (8)-(13), the derivative of $V$ is computed as

$$
\begin{aligned}
\dot{V}= & z_{1}^{T} \dot{z}_{1}+z_{2}^{T} M(q) \dot{z}_{2}+\frac{1}{2} z_{2}^{T} \dot{M}(q) z_{2} \\
& +\dot{\tilde{q}}^{T} M(q) \ddot{\tilde{q}}+\frac{1}{2} \dot{\tilde{q}}^{T} \dot{M}(q) \dot{\tilde{q}} \\
= & -z_{1}^{T} \Lambda_{1} z_{1}+z_{1}^{T} z_{2}+\frac{1}{2} z_{2}^{T} \dot{M}(q) z_{2} \\
& +\dot{\tilde{q}}^{T} M(q)(\ddot{q}-\ddot{\tilde{q}})+\frac{1}{2} \dot{\tilde{q}}^{T} \dot{M}(q) \dot{\tilde{q}} \\
& +z_{2}^{T}[\tau-C(q, \dot{q}) \dot{q}-G(q)] \\
& +z_{2}^{T}\left[-M(q)\left(\ddot{q}_{d}+\Lambda_{1}^{2} z_{1}-\Lambda_{1} z_{2}\right)\right] .
\end{aligned}
$$

From (4) in Property 2, we can obtain

$$
\begin{aligned}
\dot{V}= & -z_{1}^{T} \Lambda_{1} z_{1}+z_{1}^{T} z_{2} \\
& +z_{2}^{T}\left[\tau-C(q, \dot{q}) \dot{q}-G(q)+C(q,, \dot{q}) z_{2}\right] \\
& +z_{2}^{T}\left[-M(q)\left(\ddot{q}_{d}+\Lambda_{1}^{2} z_{1}-\Lambda_{1} z_{2}\right)\right] \\
& +\dot{\tilde{q}}^{T}[\tau-C(q, \dot{q}) \dot{q}-G(q)+C(q, \dot{q}) \dot{\tilde{q}}-M(q) \ddot{\hat{q}}] \\
= & -z_{1}^{T} \Lambda_{1} z_{1}+z_{1}^{T} z_{2}+z_{2}^{T}\left[\tau-C(q, \dot{q}) \dot{q}_{r}-G(q)\right] \\
& +z_{2}^{T}\left[-M(q)\left(\ddot{q}_{d}+\Lambda_{1}^{2} z_{1}-\Lambda_{1} z_{2}\right)\right] \\
& +\dot{\tilde{q}}^{T}[\tau-C(q, \dot{q}) \dot{\hat{q}}-G(q)-M(q) \ddot{\hat{q}}] .
\end{aligned}
$$

Now, from the designed controller (14) and observer (15), we have

$$
\begin{aligned}
\dot{V}= & -z_{1}^{T} \Lambda_{1} z_{1}-z_{2}^{T} M(q) \Lambda_{1}^{2} z_{1}-z_{2}^{T} C\left(q, \dot{q}_{r}\right) \dot{\tilde{q}} \\
& -z_{2}^{T}\left(K_{1}-M(q) \Lambda_{1}\right) z_{2}-\dot{\tilde{q}}^{T} K_{1} z_{1} \\
& -\dot{\tilde{q}}^{T} C(q, \dot{\hat{q}}) z_{2}+\dot{\tilde{q}}^{T}\left(K_{1}-M(q) L_{d}\right) \dot{\tilde{q}} . \quad(19)
\end{aligned}
$$

Note that from the (5) in Property 3, we have

$$
\begin{aligned}
& C(q, \dot{\hat{q}}) \dot{q}_{r}-C(q, \dot{q}) \dot{q}_{r} \\
& =C\left(q, \dot{q}_{r}\right) \dot{\hat{q}}-C\left(q, \dot{q}_{r}\right) \dot{q}=-C\left(q, \dot{q}_{r}\right) \dot{\tilde{q}},
\end{aligned}
$$

and

$$
\begin{aligned}
& C(q, \dot{\hat{q}}) \dot{q}_{r}-C(q, \dot{q}) \dot{\hat{q}} \\
& =C(q, \hat{q}) \dot{q}_{r}-C(q, \hat{\hat{q}}) \dot{q}=-C(q, \dot{\hat{q}}) z_{2} .
\end{aligned}
$$

By applying Property 1 and Property 4 in Section 2 , (19) becomes

$$
\begin{aligned}
\dot{V} \leq & -\lambda_{m}\left\|z_{1}\right\|^{2}-\left(k_{m}-m_{M} \lambda_{M}\right)\left\|z_{2}\right\|^{2} \\
& +m_{M} \lambda_{M}^{2}\left\|z_{1}\right\|\left\|z_{2}\right\|-\left(m_{m} l_{m}-k_{M}\right)\|\dot{\tilde{q}}\|^{2} \\
& +\|\dot{\tilde{q}}\|\left\|z_{1}\right\|+c_{M}\left\|z_{2}\right\|\|\dot{\tilde{q}}\|\left(\left\|\dot{q}_{r}\right\|+\|\dot{\hat{q}}\|\right) \cdot(22)
\end{aligned}
$$

In order to show that $\dot{V}$ can be negative definite, we employ the following analysis and investigation to verify this results. First of all, we define $\eta=$ $m_{M} \lambda_{M}^{2}$, and obtain

$$
\eta\left\|z_{1}\right\|\left\|z_{2}\right\| \leq \frac{1}{2}\left\|z_{1}\right\|^{2}+\frac{\eta^{2}}{2}\left\|z_{2}\right\|^{2},
$$

and

$$
\left\|z_{1}\right\|\|\dot{\tilde{q}}\| \leq \frac{1}{2}\left\|z_{1}\right\|^{2}+\frac{1}{2}\|\dot{\tilde{q}}\|^{2} .
$$

Since the desired velocity $\dot{q}_{d}$ is assumed to be bounded, we have

$$
\left\|\dot{q}_{d}\right\| \leq v_{M},
$$

where $v_{M}$ is a positive constant. Then, applying $\dot{q}_{r}=\dot{q}_{d}-\Lambda_{1} z_{1}$ and $\dot{\hat{q}}=z_{2}+\dot{q}_{r}-\dot{\tilde{q}}$, we have

$$
\begin{aligned}
& c_{M}\left\|z_{2}\right\|\|\dot{\tilde{q}}\|\left(\left\|\dot{q}_{r}\right\|+\|\dot{\hat{q}}\|\right) \\
& \leq c_{M}\left\|z_{2}\right\|\|\dot{\tilde{q}}\|\left(2\left\|\dot{q}_{d}\right\|+2 \lambda_{M}\left\|z_{1}\right\|+\left\|z_{2}\right\|+\|\dot{\tilde{q}}\|\right)
\end{aligned}
$$




$$
\begin{aligned}
\leq & \frac{c_{M}}{2}\left(\left\|z_{2}\right\|^{2}+\|\dot{\tilde{q}}\|^{2}\right)\left(2\left\|\dot{q}_{d}\right\|+2 \lambda_{M}\left\|z_{1}\right\|\right. \\
& \left.+\left\|z_{2}\right\|+\|\dot{\tilde{q}}\|\right) .
\end{aligned}
$$

Therefore, (22) becomes

$$
\begin{aligned}
& \dot{V} \leq-\beta_{1}\left\|z_{1}\right\|^{2} \\
& \quad+\left(-\beta_{2}+\gamma_{21}\left\|z_{1}\right\|+\gamma_{22}\left\|z_{2}\right\|+\gamma_{23}\|\dot{\tilde{q}}\|\right)\left\|z_{2}\right\|^{2} \\
& +\left(-\beta_{3}+\gamma_{31}\left\|z_{1}\right\|+\gamma_{32}\left\|z_{2}\right\|+\gamma_{33}\|\dot{\tilde{q}}\|\right)\|\dot{\tilde{q}}\|^{2}
\end{aligned}
$$

where

$$
\begin{aligned}
\beta_{1} & =\lambda_{m}-1 \\
\beta_{2} & =k_{m}-m_{M} \lambda_{M}-\frac{\eta^{2}}{2}-c_{M} v_{M} \\
\beta_{3} & =m_{m} l_{m}-k_{M}-\frac{1}{2}-c_{M} v_{M} \\
\gamma_{21} & =\gamma_{31}=c_{M} \lambda_{M} \\
\gamma_{22} & =\gamma_{32}=\frac{c_{M}}{2} \\
\gamma_{23} & =\gamma_{33}=\frac{c_{M}}{2} .
\end{aligned}
$$

Furthermore, (28) can be rewritten as follows:

$$
\begin{aligned}
\dot{V} \leq & -\beta_{1}\left\|z_{1}\right\|^{2} \\
& -\sum_{i=2}^{3}\left(\beta_{i}-\sum_{j=1}^{3} \gamma_{i j}\left\|\chi_{j}\right\|\right)\left\|\chi_{i}\right\| .
\end{aligned}
$$

From the Lemma introduced in Appendix, if we select the following conditions:

- $\lambda_{m}>1$,

$$
\begin{aligned}
& \text { - } k_{m}>m_{M} \lambda_{M}+\frac{\eta^{2}}{2}+c_{M} v_{M}+\sum_{j=1}^{3}\left(\frac{V_{0}}{\xi_{j}}\right)^{\frac{1}{2}}, \\
& \text { - } m_{m} l_{m}>k_{M}+\frac{1}{2}+c_{M} v_{M}+\sum_{j=1}^{3}\left(\frac{V_{0}}{\xi_{j}}\right)^{\frac{1}{2}},
\end{aligned}
$$

where

$$
\xi_{1}=\frac{1}{2} k_{m}, \quad \xi_{2}=\frac{1}{2} m_{m}, \quad \xi_{3}=\frac{1}{2} m_{m},
$$

and

$$
V_{0} \equiv V\left(z_{1}(0), z_{2}(0), \dot{\tilde{q}}(0)\right) .
$$

The following inequality holds

$$
\begin{aligned}
\dot{V} & \leq-\beta_{1}\left\|z_{1}\right\|^{2}-\lambda_{2}\left\|z_{2}\right\|^{2}-\lambda_{3}\|\dot{\tilde{q}}\|^{2}<0 \\
\forall \lambda_{i} & \in\left[0, \beta_{i}-\sum_{j=1}^{3} \gamma_{i j}\left(\frac{V_{0}}{\xi_{j}}\right)^{\frac{1}{2}}\right],(i=2,3) .
\end{aligned}
$$

As a result, we can conclude that $\dot{V}$ is negative definite. From (16) and (29), it implies that the resulting closed-loop system is exponentially stable according to Lyapunov stability theorem. In other words, not only the stability of the system can be guaranteed, but also that the trajectory tracking error and angular velocity estimation
Table 1. System parameter values of the two-link robot manipulator model.

\begin{tabular}{cccc}
\hline Parameter & Value & Parameter & Value \\
\hline$m_{1}$ & $5 \mathrm{~kg}$ & $\mathrm{~m}_{2}$ & $5 \mathrm{~kg}$ \\
$l_{1}$ & $0.5 \mathrm{~m}$ & $l_{2}$ & $0.5 \mathrm{~m}$ \\
$l_{c 1}$ & $0.25 \mathrm{~m}$ & $l_{c 2}$ & $0.25 \mathrm{~m}$ \\
$I_{1}$ & $0.104 \mathrm{~kg} \cdot \mathrm{m}^{2}$ & $I_{2}$ & $0.104 \mathrm{~kg} \cdot \mathrm{m}^{2}$ \\
\hline
\end{tabular}

error converge to zero finally. It can be shown that the proposed control scheme (14) guarantees exponential stability for the tracking control of robot manipulator with only position measurement. The velocity observer (15) can estimate the unknown angular velocity of each joint of robot manipulator.

\section{SIMULATION RESULTS}

In our simulations, a two-link robot manipulator is considered with the masses $m_{1}$ and $m_{2}$, the lengths $l_{1}$ and $l_{2}$, the angles $q_{1}$ and $q_{2}$, the moments of inertia $I_{1}$ and $I_{2}$, and the control torques $\tau_{1}$ and $\tau_{2}$. In addition, $l_{c 1}$ and $l_{c 2}$ are the distances from the center of mass to the joint axes. The dynamic model of a two-link robot manipulator can be written as follows:

$\left[\begin{array}{l}\tau_{1} \\ \tau_{2}\end{array}\right]=\left[\begin{array}{ll}M_{1} & M_{2} \\ M_{3} & M_{4}\end{array}\right]\left[\begin{array}{l}\ddot{q}_{1} \\ \ddot{q}_{2}\end{array}\right]+\left[\begin{array}{ll}C_{1} & C_{2} \\ C_{3} & C_{4}\end{array}\right]\left[\begin{array}{l}\dot{q}_{1} \\ \dot{q}_{2}\end{array}\right]+\left[\begin{array}{l}G_{1} \\ G_{2}\end{array}\right]$,

where

$$
\begin{aligned}
& M_{1}=m_{1} l_{c 1}^{2}+I_{1}+m_{2}\left(l_{1}^{2}+l_{c 2}^{2}+2 l_{1} l_{c 2} \cos q_{2}\right)+I_{2} \\
& M_{2}=M_{3}=m_{2} l_{1} l_{c 2} \cos q_{2}+m_{2} l_{c 2}^{2}+I_{2} \\
& M_{4}=m_{2} l_{c 2}^{2}+I_{2} \\
& C_{1}=-m_{2} l_{1} l_{c 2} \sin q_{2} \dot{q}_{2} \\
& C_{2}=-m_{2} l_{1} l_{c 2} \sin q_{2}\left(\dot{q}_{1}+\dot{q}_{2}\right) \\
& C_{3}=m_{2} l_{1} l_{c 2} \sin q_{2} \dot{q}_{1} \\
& C_{4}=0 \\
& G_{1}=m_{1} g l_{c 1} \cos q_{1}+m_{2} g\left(l_{1} \cos q_{1}+l_{c 2} \cos \left(q_{1}+q_{2}\right)\right) \\
& G_{2}=m_{2} g l_{c 2} \cos \left(q_{1}+q_{2}\right) .
\end{aligned}
$$

The system parameters of this two-link of robot manipulator model are selected as Table 1 . In addition, the reference trajectories are given as the following two cases:

- Case 1: $q_{d 1}=q_{d 2}=1.25-1.25 e^{-t}$, and

- Case 2: $q_{d 1}=q_{d 2}=\sin t$.

The design constants for the nonlinear controller (14) and observer (15) are selected as

$\Lambda_{1}=\left[\begin{array}{ll}2 & 0 \\ 0 & 2\end{array}\right], \quad K_{1}=\left[\begin{array}{rr}30 & 0 \\ 0 & 30\end{array}\right], \quad L_{d}=\left[\begin{array}{rr}30 & 0 \\ 0 & 30\end{array}\right]$.

Figs. 1 and 2 show the simulation results with the reference trajectory in Case 1, and Figs. 3 and 4 are the simulation results with the reference trajectory in Case 2, which contains periodic 
trajectories. The plots of the trajectories of joint 1 and joint 2 are shown in Figs. 1 and 3, where the desired trajectory of each joint is shown with dotted line, and the actual position of each joint is plotted with solid line. The velocity estimation errors of joint 1 and joint 2 are shown in Figs. 2 and 4 with different cases of reference signals.

All of these simulation results illustrate excellent performance of the proposed nonlinear controller with the given observer. The simulation plots shown in Figs. 1 and 3 indeed verify that our nonlinear backstepping design scheme can guarantee each joint of the robotic manipulator to follow its desired trajectory exponentially. Figs. 2 and 4 show the velocity estimation error between the actual system velocity and the estimated velocity. From these figures, we can find out that all the velocity estimation errors converge to zero exponentially. It implies that the estimated velocity of each joint converges to its actual velocity rapidly.

\section{CONCLUDING REMARKS}

In this paper, a nonlinear backstepping design method has been proposed for the control of robot manipulators with velocity observers to achieve the desired trajectory tracking control objectives. Hence, the movement of robot manipulators can be controlled with only position measurements. The resulting closed-loop system is exponentially stable, that is, the trajectory tracking errors and velocity estimation errors converge to zero exponentially according to Lyapunov stability theorem. With this backstepping design approach, the simulation results of a two-link robot manipulator are seemed to be excellent for the trajectory tracking control with velocity estimation.

Besides the angular velocity observation, if system dynamic uncertainties and load disturbances of the robot manipulator are existent, then their estimations must be considered in order to enhance the system performance. Therefore, adaptive backstepping control scheme should be applied for the tracking control design of robotic manipulator systems under these circumstances in the further research.

\section{APPENDIX}

An important stability lemma mentioned below plays a major role for the system stability analysis in this paper. This Lemma is a local stability theorem that establishes a region of convergence. It will be shown that if the initial state is within some ball of radius $\beta$, then the state never escape the $\beta$-ball.

Lemma (Wen and Bayard, 1988): Consider a
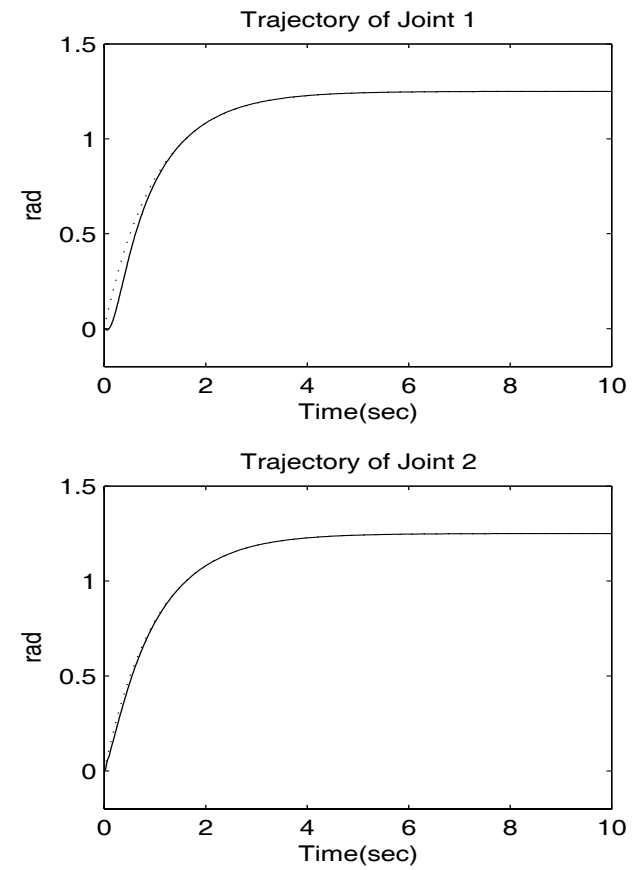

Fig. 1. The trajectories of the two-link robot manipulator (Case 1): desired trajectory (... dotted line) vs. actual position (- solid line).
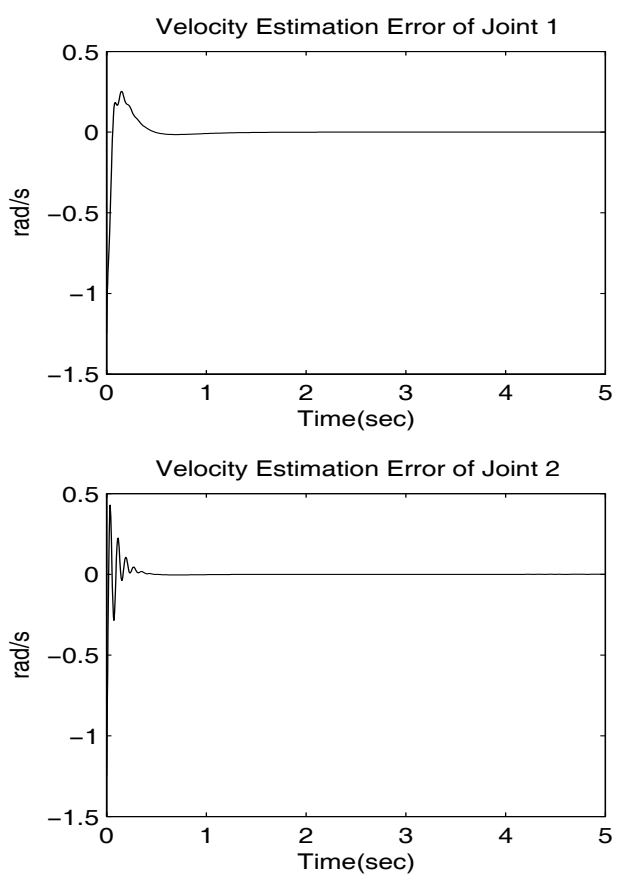

Fig. 2. The velocity estimation error of the twolink robot manipulator (Case 1)

dynamic system $\dot{x}_{i}=f_{i}\left(x_{i}, \ldots, x_{N}, t\right), x_{i} \in R^{n}$, $t \geq 0, i=1, \ldots, N$, where $f_{i}$ is locally Lipschitz with respect to $x_{1}, \ldots, x_{N}$, uniformly in $t$ on bounded intervals and continuous in $t$ for $t \geq 0$. Suppose that a function $V$ is given such that

$$
V\left(x_{1}, \ldots, x_{m}\right)=\sum_{i, j=1}^{m} x_{i}^{T} P_{i j} x_{j},
$$

and for $i=1, \ldots, m$, there exists $\xi_{i}>0$ such that 

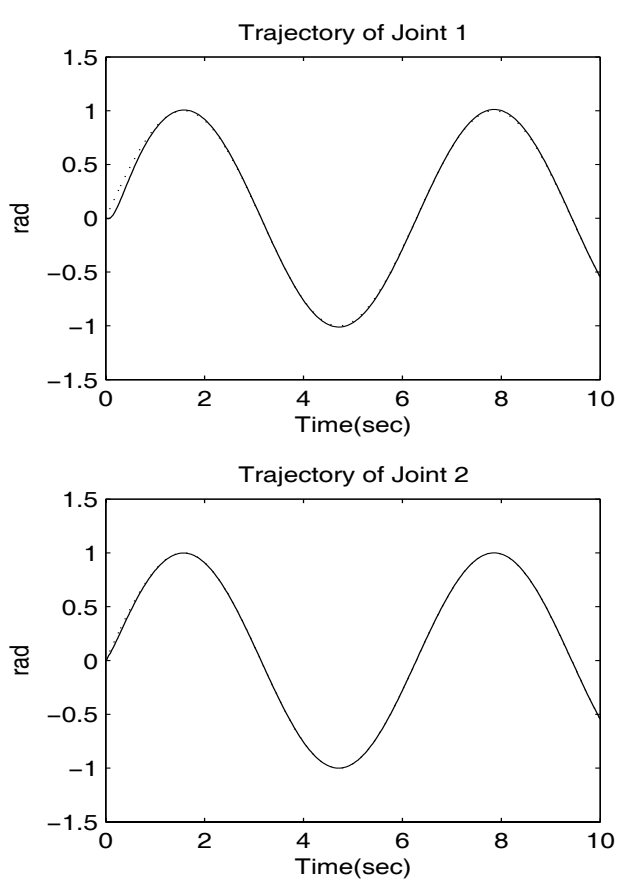

Fig. 3. The trajectories of the two-link robot manipulator (Case 2): desired trajectory (... dotted line) vs. actual position (- solid line).
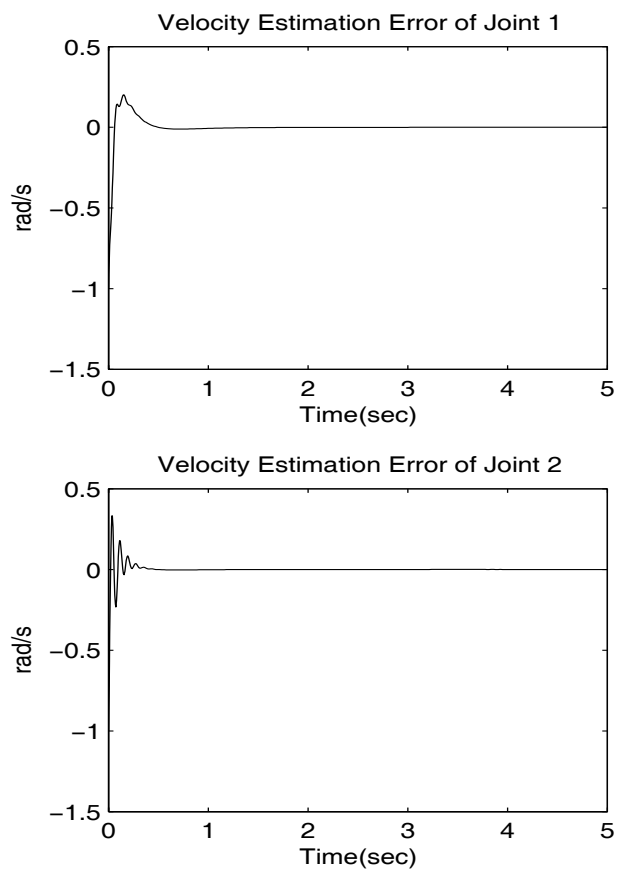

Fig. 4. The velocity estimation error of the twolink robot manipulator (Case 2)

$$
\begin{aligned}
\xi_{i}\left\|x_{i}\right\|^{2} & \leq V\left(x_{1}, \ldots, x_{m}\right) \\
\dot{V}\left(x_{1}, \ldots, x_{m}\right) & \leq-\sum_{i \in I_{1}}\left(\beta_{i}-\sum_{i \in I_{2}} \gamma_{i j}\left\|x_{j}\right\|\right)\left\|x_{i}\right\|^{2},
\end{aligned}
$$

where $\beta_{i}>0, \gamma_{i j}>0$ and $I_{2} \subset I_{1} \subset\{1, \ldots, m\}$. Let $V_{0} \equiv V\left(x_{1}(0), \ldots, x_{m}(0)\right)$. If for all $i \in I_{1}$,

$$
\beta_{i}>\sum_{j \in I_{2}} \gamma_{i j}\left(\frac{V_{0}}{\xi_{j}}\right)^{\frac{1}{2}}
$$

then $\forall \lambda_{i} \in\left[0, \beta_{i}-\sum_{j \in I_{2}} \gamma_{i j}\left(\frac{V_{0}}{\xi_{j}}\right)^{\frac{1}{2}}\right]$, the following inequality holds

$$
\dot{V}\left(x_{1}, \ldots, x_{m}\right) \leq-\sum_{i \in I_{1}} \lambda_{i}\left\|x_{i}\right\|^{2}, \quad \forall t \geq 0 .
$$

It implies that the dynamic system is locally exponentially stable according to Lyapunov stability theorem.

\section{REFERENCES}

Berghuis, H. and H. Nijmeijer (1991). Tracking control of robots using only position measurement. In: Proceedings of the 30th Conference on Decislon and Control. Vol. 2. pp. 10391040. Brighton, England.

Calugi, F., A. Robertsson and R. Johansson (2002). Output feedback adaptive control of robot manipulators using observer backstepping. In: Proceedings of the 2002 IEEE International Conference on Intelligent Robots and Systems. pp. 2091-2096.

Craig, J.J. (1988). Adaptive Control of Mechanical Manipulators. Addison-Wesley.

Eric, M. and W.S. Lu (1996). A reduced-order adaptive velocity observer for manipulator control. IEEE Transactions on Robotics and Automation 11, 293-303.

Khalil, H.K. (2002). Nonlinear Systems. 3rd ed.. Prentice-Hall. Upper Saddle River, NJ.

Krstić, M., I. Kanellakopoulos, and P. Kokotović (1995). Nonlinear and Adaptive Control Design. Wiley-Interscience.

Lim, S.Y., D.M. Dawson and K. Anderson (1996). Re-examining the nicosia-tomei robot observer-controller from a backstepping perspective. IEEE Transactions on Control Systems Technology 4, 304-310.

Lin, J.-S. and C.-J. Huang (2003). Nonlinear backstepping control desdign of half-car active suspension systems. Internatonal Journal of Vehicle Design 33, 332-350.

Nisosia, S. and P. Tomei (1990). Robot control by using joint position measurements. IEEE Transactions on Automatic Control 35, 10581061.

Ting, W.-E. and J.-S. Lin (2004). Nonlinear control design of anti-lock braking systems combined with active suspensions. In: Proceeding of the 5th Asian Control Conference. pp. 610615. Melbourne, Australia.

Tsai, F.-K. and J.-S. Lin (2003). Nonlinear control design of 360-degree inverted pendulum systems. In: Proceedings of the 4 th International Conference on Control and Automation. pp. 634-638. Montreal, Canada.

Wen, J.T. and D.S. Bayard (1988). New class of control laws for robotic manipulators: nonadaptive case.. International Journal of Control 47, 1361-1385. 